\title{
Correction to: A thiotrophic microbial community in an acidic brine lake in Northern Chile
}

\author{
Lorena Escudero - Nia Oetiker - Karem Gallardo - Cinthya Tebes-Cayo • \\ Mariela Guajardo • Claudia Nuñez - Carol Davis-Belmar • J. J. Pueyo • \\ Guillermo Chong Díaz $\cdot$ Cecilia Demergasso $\mathbb{D}$
}

Published online: 3 September 2018

(C) Springer Nature Switzerland AG 2018

Correction to: Antonie van Leeuwenhoek (2018) 111:1403-1419 https://doi.org/10.1007/s10482-018-1087-8

In Table 1 of the original article, the unit $\mathrm{mg} / \mathrm{L}$ was incorrectly published as $\mathrm{ng} / \mathrm{L}$ in the aluminum, chloride, sulphate and OM columns.

The correct Table 1 is provided below,

The original article can be found online at https:// doi.org/10.1007/s10482-018-1087-8.

L. Escudero $\cdot$ N. Oetiker $\cdot$ K. Gallardo ·

C. Tebes-Cayo · C. Nuñez · C. Demergasso $(\square)$

Centro de Biotecnología, Universidad Católica del Norte,

Antofagasta, Chile

e-mail: cdemerga@ucn.cl

L. Escudero · M. Guajardo · C. Demergasso

Centro de Investigación Científica y Tecnológica para la

Minería, Antofagasta, Chile

C. Davis-Belmar

BHP, Cerro El Plomo 6000, Las Condes,

PO Box 7560623, Santiago, Chile
G. C. Díaz

Departamento de Geología, Universidad Católica del Norte, Antofagasta, Chile

J. J. Pueyo

Departament de Geoquímica, Facultat de Geología, Universitat de Barcelona, Barcelona, Spain 


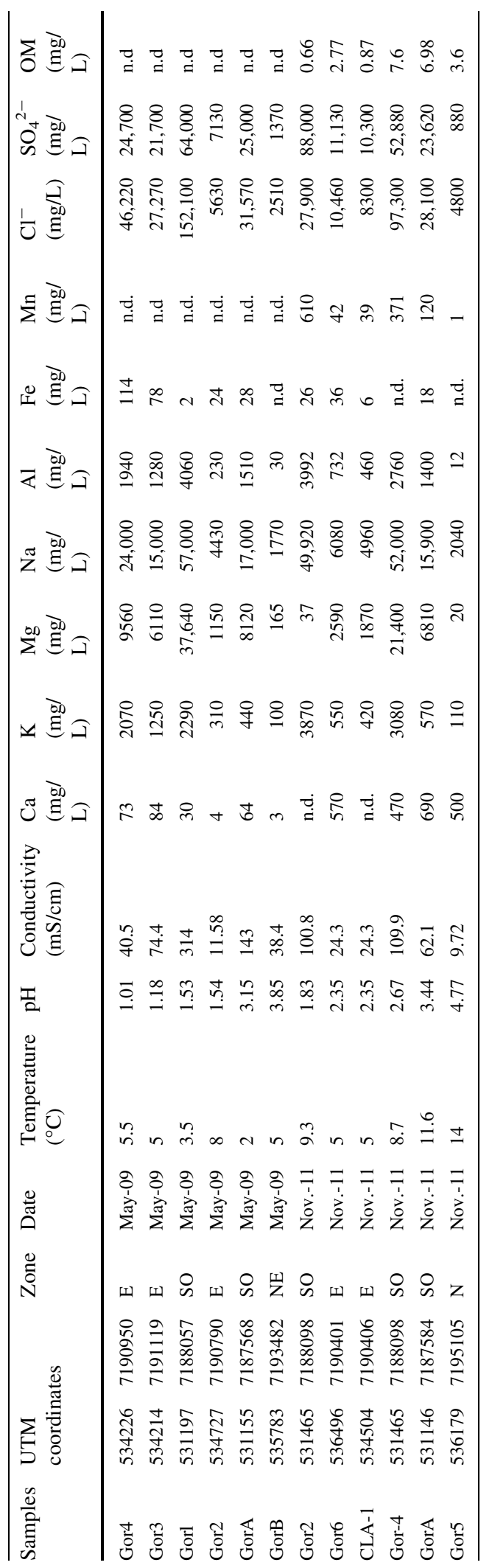

